“C 2018 IEEE. Personal use of this material is permitted. Permission from IEEE must be obtained for all other uses, in any current or future media, including reprinting/republishing this material for advertising or promotional purposes, creating new collective works, for resale or redistribution to servers or lists, or reuse of any copyrighted component of this work in other works." 


\title{
Performance Analysis of Full Duplex D2D in Opportunistic Spectrum Access
}

\author{
Noman Haider ${ }^{1}$, Ahsan $\mathrm{Ali}^{2}$, Ying $\mathrm{He}^{1}$, and Eryk Dutkiewicz ${ }^{1}$ \\ ${ }^{1}$ University of Technology Sydney, Global Big Data Technology Centre, Ultimo NSW 2007, Australia \\ ${ }^{2}$ Department of Engineering, Macquarie University, Australia.
}

\begin{abstract}
Opportunistic Spectrum Access (OSA) allows an efficient use of spectrum based on share-it or use-it principle and can be a viable solution for the challenging problem of spectrum scarcity. Emerging systems have been proposed for OSA, where primary users (PU) have guaranteed interference protection from secondary users (SU). The potential of Full Duplex (FD) and Device-to-device (D2D) technologies in 5G has proven to be promising for increasing data rates and network capacity. In this article using stochastic geometry and random graphs, we model and assess the D2D operations in full Duplex/half Duplex mode for SUs, while protecting the PU's transmission by defining the exclusion zone (EZ). Depending on the location and transmit power of D2D users, the induced aggregate interference should not violate the interference threshold for EZ of PUs. For this, we characterize the interference from D2D links and derive the probability for successful D2D users for half-duplex and full duplex modes. Analyses is further supported by extensive simulations results.
\end{abstract}

Index Terms-Opportunistic Spectrum Access (OSA), Stochastic geometry, Full Duplex, Device-to-device (D2D), Poisson Point process (PPP), Poisson Hole process (PHP), Exclusion Zone (EZ).

\section{INTRODUCTION}

Spectrum is the key resource for remote and wireless connectivity of devices to Internet. Due to the gigantic increase in the number of connected devices, spectrum scarcity has proven to be a challenging research problem in recent years. The demand for ubiquitous connectivity and high data rates have motivated network providers and vendors to come up with an optimum use of existing resources (spectrum) and integration of new technologies (Full Duplex, D2D). The development and testing of such solutions is also one of the driving factors for future generation $(5 \mathrm{G})$ of mobile networks. Among these proposals, Cognitive radio, TV white spaces (TVWS), Citizen Broadband Radio Service (CBRS), Spectrum Access System (SAS), Licensed Shared Access (LSA) and multi Radio Access Technologies (RATs) have proven to be an effective solutions for spectrum scarcity.

The key idea behind spectrum sharing is the use-it or shareit rule, where primary licensed users can share underutilized spectrum with secondary unlicensed users conditioned on interference protection from secondary users. This sharing is done based on pre-defined conditions for leaving the spectrum for priority users whenever needed and imposing the least interference to primary users. Spectrum sharing can be done in the time domain (primary user is not transmitting), space domain (primary user is far away) and frequency domain (primary user is transmitting on a different frequency). For detailed benefits of dynamic spectrum sharing and heterogeneous device coexistence, readers are referred to [1].

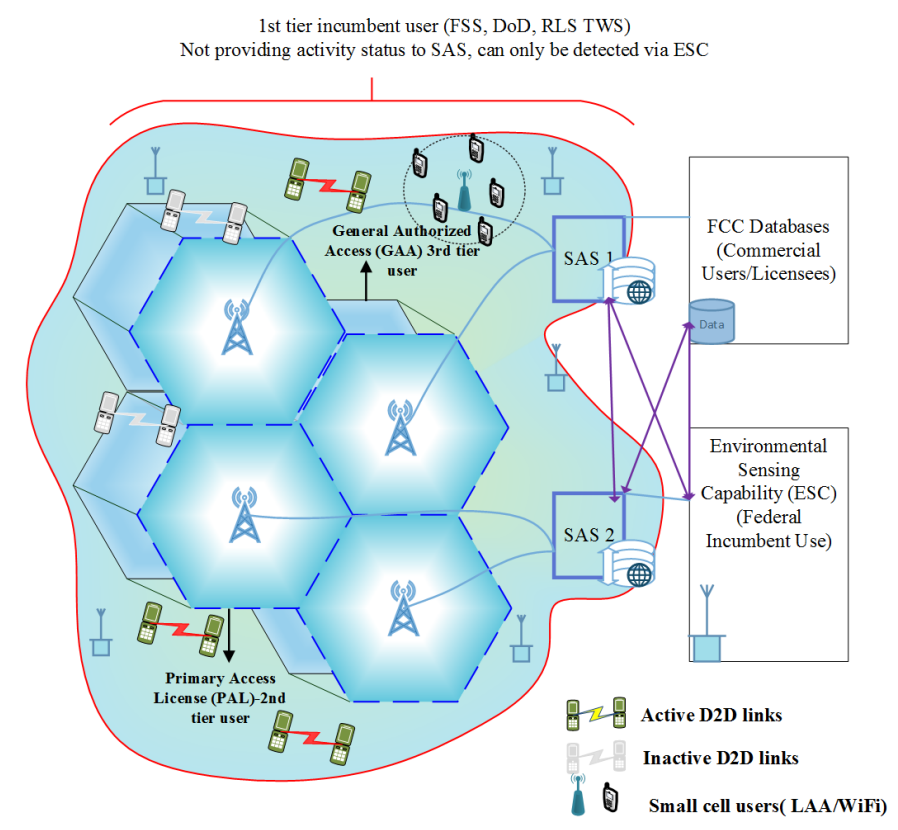

Fig. 1: A typical illustration of considered CBRS system with incumbents (tier-1 user), priority access licensed (tier-2 cellular) and general authorized access (D2D and small cells tier-3) users.

The D2D communication has significantly shown its potential to elevate the user experience and efficiently improve the network capacity by traffic off-loading from the main network. It is also one of the key enabling technologies in future wireless and cellular networks [2]. D2D is a good technology candidate for opportunistic dynamic spectrum sharing without producing harmful aggregate interference to other devices (due to shorter link distances and lower transmit powers). In this article, we propose D2D technology as a tier-2 (SU) technology candidate and model the system by characterizing the interference and success probability [3]. Due to strict interference threshold conditions which SU has to comply with for PU transmission protection, D2D has more potential as compared to LTE-LAA and WiFi as the D2D users can 
communicate in a near distance of Exclusion Zones (EZ). The D2D network has performance advantages as compared to other small cell technologies due to limited interference and near-distant communication between transmitters (TXs) and receivers (RXs). For instance, a CBRS system and active/inactive D2D links based on their location and proximity to EZ of PU is shown in Fig. 1.

The rest of the paper is organized as follows. The related work is presented in section II. The detailed system model, problem addressed, propagation assumptions and received power expressions are provided in Section III. Then, we derive an expression for probability of successful transmission for a typical D2D link in section IV. Simulation results and discussion are the presented in section V. Finally, the conclusion of the paper is presented in Section VI.

\section{RELATED WORK}

Stochastic Geometry (SG) and random graph theory have proven to be an effective mathematical platform to model variants of communication networks while characterizing the key network parameters [4]. Due to topological and spatial randomness, SG can successfully yield tractable and in special cases, closed form expressions that reflect the system behavior. The alternate methods for performance evaluation of cellular networks include exhaustive simulation scenarios to average out randomness of different network parameters (BS, user locations and fading distributions). However, they are timeconsuming and prone to errors. Therefore, SG provides a supplementary platform to produce baseline results for benchmarking and comparison [5].

In the context of dynamic spectrum sharing, recently stochastic geometry modeling and analysis of CBRS is done in [3]. Authors present tractable performance analysis of Spectrum Access System (SAS) by employing EZ for priority access licensed (PAL) users, while general authorized access (GAA) users operate using contention based channel access mechanism (CSMA). Previously, similar work has been done in the context of cognitive radio where opportunistic spectrum access is exploited. However, due to unique interference restrictions in EZ of PAL and induced aggregate interference from GAA users makes CBRS systems challenging. For instance, authors in [6] presented a stochastic geometry model to characterize interference from SU to PUs and also in multitier networks. Similar approach of employing EZ has been used in [7], SG analysis for co-existence of contention-based (WiFi) and scheduled based (LTE) networks is presented in [8]. Moreover, the SG analysis for interference characterization and expressions for network performance metrics for $\mathrm{K}$-tier heterogeneous cellular networks is presented in [9]. Most of the analysis in this domain uses Matern hardcore process of type-II (MHPP-II) [10] to study the coexistence among licensed (primary) and unlicensed (secondary) users, which are limited to only bi-polar networks. However, due to strict interference limitations on EZ boundaries of PAL, CBRS requires sophisticated analysis to locate operational zones for GAA's operations. Another work in [11] investigate the impact of different coexistence techniques for FD D2D with cellular and WiFi. This article analyzes the opportunistic use of D2D devices in vicinity of EZ's while limiting the induced interference to protect PALs. SG analysis of FD D2D has also been recently studied and performance trade-off have been studied [12]. The initial SG analysis for FD gains were presented in [13]. Another SG approach presented signal to interference and noise ratio (SINR), transmit-power and mode switching (HD/FD) for FD D2D for cellular networks [14]. Interestingly, a similar concept has recently been proposed to use OSA for Machine-Type Communications [15]. In this article, performance analysis of a second technology candidate can be equated to use of D2D, machine-type devices and similar stationary users.

\section{SYSTEM MODEL}

\section{A. Spatial locations}

We consider a cognitive system (three-tier), where PU operate on leased spectrum of incumbents and are licensed to use this spectrum in absence of incumbents. The leased spectrum is segregated into small chunks, we assume PU is operating on one of these selected frequency band. Moreover, we consider the up-link only transmission between transmitter (TXs) and Receiver (RXs). The second-tier D2D enabled SU network can opportunistically share incumbents spectrum outside of the exclusion zone while abiding by the interference thresholds for protection of PU transmissions. To model the locations of D2D receivers we assume that all the nodes are uniformly distributed in $\mathbb{R}^{2}$ and takes on a realization of a Poisson point process (PPP) with intensity $\lambda_{D}$. We denote this point process with $\Phi_{D}$, and $y$ to denote a point in $\Phi_{D}$, i.e., $y \in \Phi_{D}$. For each point $y \in \Phi_{D}$ we assign a mark $m(y)$ which is uniformly randomly distributed on a circle of radius $R_{D 2 D}$.

To model the protection of cellular user uplink transmission, we assume a circle $\mathcal{C}$ of radius $R_{E Z}$ located at distance $s$ from origin. All the D2D users that falls within circle $\mathcal{C}$ are not allowed to transmit in order to protect the uplink transmission of cellular user. We represent the D2D users which are allowed to transmit out of the EZ by $\Psi_{D}$ with intensity given as follows:

$$
\tilde{\lambda}_{D}=\lambda_{D} \exp \left(-\pi \lambda_{C} R_{E Z}^{2}\right),
$$

where $\lambda_{C}$ denoted the intensity of cellular users.

To model the state of D2D links, i.e., HD or FD, we assign another mark $s(y)$ for each point $y \in \Psi_{D}$. These marks represents the probability that whether a specific link can operate in FD or HD.

Only the D2D pairs outside of PU EZ are authorized to communicate if they meet the IP conditions. The D2D communication pair is assigned an independently chosen link state with probability $p_{H D}$ or $p_{F D}$ such that $p_{H D}+p_{F D}=1$. The self interference leakage in FD links is considered to be imperfect with a residual self-interference-to-power-ratio (SIPR) $\beta$. The value of $\beta$ ranges from 0 to 1 for perfect and 


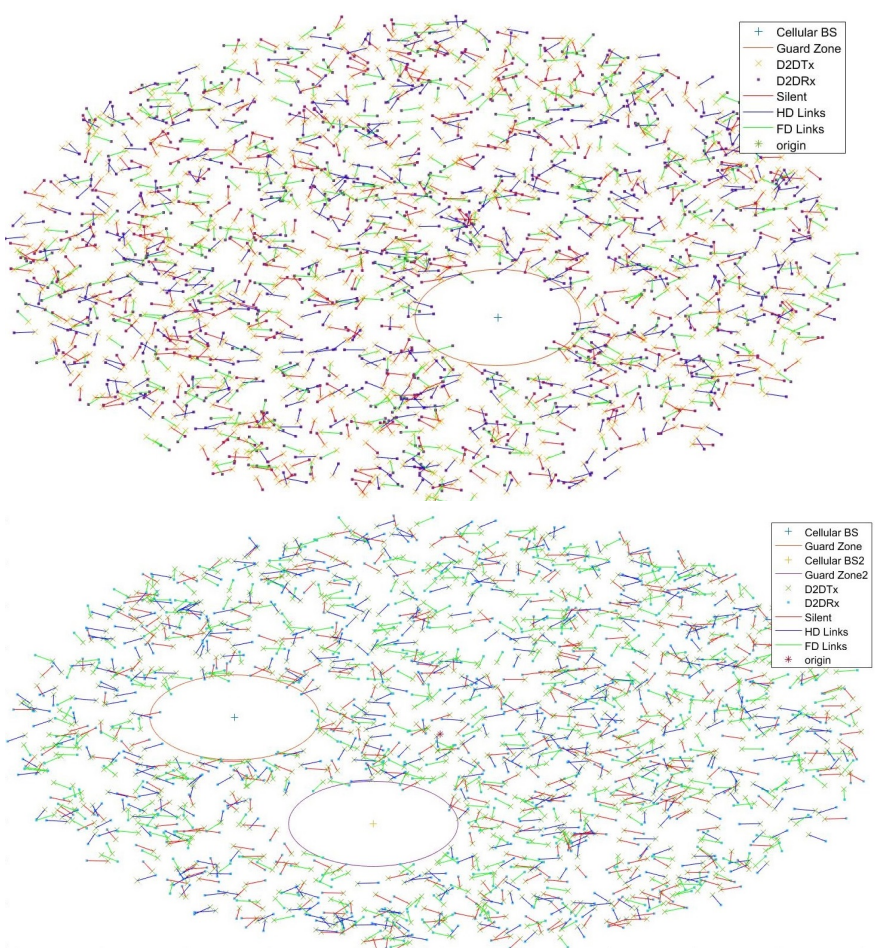

Fig. 2: Realization of network model with cellular exclusion zones and D2D Links (silent, HD and FD mode) with one EZ and two non-overlapping EZ.

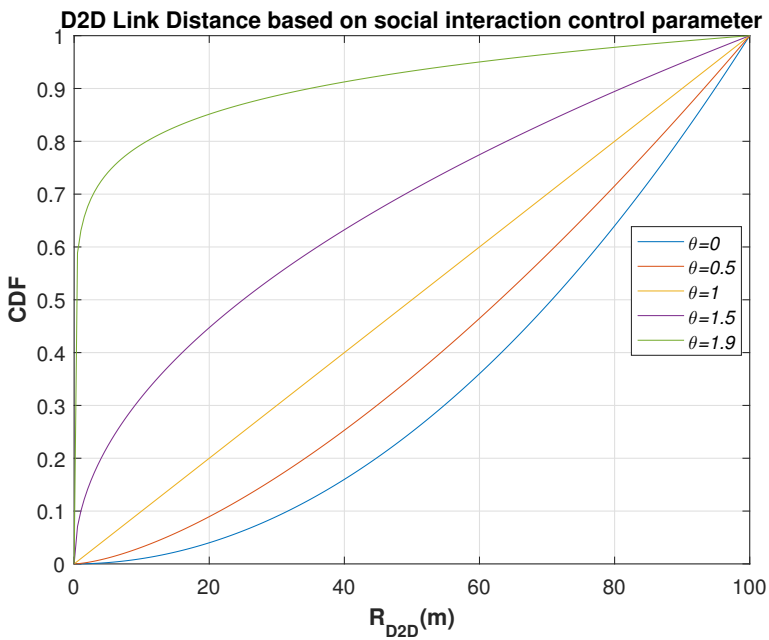

Fig. 3: CDF of D2D Link Distance for different values of $\theta$ (control parameter for social interaction)

imperfect SIPR, respectively. A simple realization of system described above is shown in Fig. 2.

The probability density function (PDF) of the contact distance distribution of homogeneous PPP is, $f_{r}=\lambda_{P} 2 \pi r e^{\left(-\lambda_{P} \pi r^{2}\right)}, r>0$. The distribution of first, second and $n_{t h}$ user in the PPP distribution can be found in [16]. The accurate contact distance distribution of PHP requires consideration of overlapping among $\mathrm{EZ}$ and spatial dependency of parent process, which makes it a NP hard problem. In context of a D2D user distribution, it depends on the underlying application and social interactions among the users. One of the trivial distance distributions for D2D users is formulated in [17], based on power-law communication probability $(0 \leq \theta<2)$ the PDF of social communication distance $D_{s}$ is given by

$$
f_{D_{s}}(v)=\frac{(2-\theta) v^{1-\theta}}{r_{v, m(y)}^{2-\theta}}
$$

here, $D_{s}$ is Random Variable (RV) representing D2D link distance. $r_{v, m(y)}$ is the maximum transmission range and $\theta$ is the control parameter for contact distance distribution (depends on social interaction of D2D users). CDF for different D2D link distances and $\theta$ values is shown in Fig. 3.

\section{B. Propagation assumptions}

Random wireless channel effects are taken into account for this analysis as well. In this regard we assume that each link in a wireless network described above experience a Rayleigh fading $F$ with mean 1 and can be modeled via exponential random variable i.e., $F \sim \exp (1)$. For large scale fading we assume a distance based path loss model with pathloss exponent $\alpha=\{2,4\}$. As cellular links and D2D links experience different propagation conditions so we use $\alpha_{C}$ and $\alpha_{D}$ for cellular and D2D users respectively. Each cellular and D2D transmitter transmits with a power of $P_{D}$ and $P_{C}$ respectively.

Without loss of generality we can assume that our test receiver is located at origin which is permissible due to Slivnyak's theorem for PPP [18]. The received useful power at test receiver can now be represented as:

$$
P_{r}\left(y_{o}\right)=P_{D} F_{o, m(o)}^{D} l(o, m(o)),
$$

where $l(o, m(o))$ represents the distance based path loss which is given by $l(o, m(o))=\|o-m(o)\|^{-\alpha_{D}}$, and $\|$.$\| is Euclidean$ norm operator. The conclusions drawn from the analysis of system model described above is equally applicable to all nodes in the network due to stationarity of PPP. Symbols, definitions and corresponding simulation values are listed in Table I.

\section{Success Probability}

The process $\Phi_{D}$ is PPP, while $\Psi_{D}$ and $\dot{\Psi_{D}}$ are close approximations to PHP due to the introduction of EZ for cellular users. Such spatial dependency of a thinned process yields analytical complexity and may not result in tractable results. Thus, in literature, such correlations among point processes are approximated to either PPP or PHP (with upper and lower bounds). Similar approximations are adopted in conventional analysis and proven to be accurate. Readers are referred to [14] for comparative analysis of distribution approximations and final results. 
TABLE I: List of Notations, Symbols and Simulation values

\begin{tabular}{|c|c|c|}
\hline Notation & Description & Simulation Value \\
\hline$\Phi_{C}, x_{i}, \lambda_{C}$ & $\begin{array}{l}\text { Homogeneous and independent PPP modeling of cellular users, ground } \\
\text { PPP and intensity of } \Phi_{C}\end{array}$ & $\{0.32,0.096,0.20,0.29,0.40\} U$ sers $/ K^{2} m^{2}$ \\
\hline$\Psi_{D}, y_{i}, \dot{\lambda_{D}}$ & $\begin{array}{l}\text { Poisson Hole Process (PHP) modeling of D2D receivers, ground PPP and } \\
\text { intensity of } \Psi_{D}\end{array}$ & $\{0.003,0.009,0.05,0.1\} U$ sers $/ K^{2}$ \\
\hline$\Psi_{m(D)}, m(y), \lambda_{m(D)}$ & $\begin{array}{l}\text { Poisson Hole Process (PHP) modeling of D2D receivers, ground PPP and } \\
\text { intensity of } \Psi_{D}\end{array}$ & $\dot{\lambda_{D}}$ \\
\hline$R_{E Z}$ & Exclusion zone radius & $\{10,20,40,60,80\} m$ \\
\hline$\alpha_{C}, \alpha_{D}$ & Path-loss component for cellular and D2D & 4,3 \\
\hline $\mathbf{E}_{1 F D}=p_{i}$ & State of D2D communication Link with probability $p_{H D / F D}$ & $\left\{p_{F D}=0,0.3,0.5,0.9\right\}$ \\
\hline $\mathcal{C}\left(s, R_{E Z}\right), \mathcal{C}^{o}\left(s, R_{E Z}\right)$ & Closed (open) ball with center $\mathrm{s}$ and radius $R_{E Z}$ & $R_{E Z}$ \\
\hline$F_{o, i}^{D}$ & Fading of channel from ith to tagged user at origin $(o, o)$ & $\mu$ \\
\hline$P_{C}, P_{D}$ & Transmit power for cellular and D2D nodes & $1,0.05$ \\
\hline$\beta$ & Residual self-interference-to-power ratio (SIPR)for FD nodes & 0.3 \\
\hline$T$ & SIR threshold for successful communication & $-20: 1: 20$ \\
\hline
\end{tabular}

The set of interfering field (intensity of interfering users) constitutes active D2D users in HD/FD mode and active cellular users, represented as:

$$
\lambda_{I F}=p_{F D} \tilde{\lambda}_{D} 1_{y, m(y)}^{F D} \cap p_{H D} \tilde{\lambda}_{D} \cap \mathcal{C}^{c}\left(s, R_{E Z}\right) \cap \lambda_{C}
$$

When a D2D link is in the FD mode, induced interference from the D2D receivers also adds up to aggregate interference to a typical receiver and constitutes PHP of intensity $\dot{\lambda_{G}}$. That's a trade-off/cost of FD operation at the benefit of increased capacity/data rate. Considering this system model and a typical receiver with a tagged transmitter at the origin, the Signal to Interference Ratio (SIR) for cellular and D2D receivers can be written respectively as:

$$
\begin{gathered}
S I R_{o} C=\frac{P_{C} F_{o, x_{o}}^{D} l\left(o, x_{o}\right)}{\mathcal{I}_{o, x / x_{o}}+\mathcal{I}_{o, y}+\mathcal{I}_{o, m(y)}+\beta P_{D} 1_{y, m(y)}^{F D}}, \\
S I R_{y_{o}^{D}}=\frac{P_{D} F_{y_{o}, m_{y_{o}}}^{D} l\left(y_{o}, m_{y_{o}}\right)}{\mathcal{I}_{y_{o}, x}+\mathcal{I}_{y_{o}, y}+\mathcal{I}_{y_{o}, m}(y) / m_{y_{o}}+\beta P_{D} 1_{y, m(y)}^{F D}},
\end{gathered}
$$

where

$$
\mathcal{I}_{y_{o}, x}=\sum_{x \in \Phi_{x}} P_{P} F_{y_{o}, x}^{P} l(0, x)
$$

is interference received at a typical user from cellular users. This interference will be higher for more BS and cellular users according to their distribution. The second interference term represents interference at a typical user from all the other D2D receivers (operating in the FD mode with probability $p_{F D}$ ).

$$
\mathcal{I}_{y_{o}, y}=\sum_{y \in \Phi_{y} / y_{o}} P_{D} F_{y_{o}, y}^{D} l\left(y_{o}, y\right) 1_{y, m(y)}^{F D}
$$

Similarly, the interference from D2D transmitters in vicinity of a typical receiver is represented by

$$
\mathcal{I}_{y_{o}, m(y)}=\sum_{m(y) \epsilon \Phi_{y} / m(0)} P_{D} F_{y_{o}, m(y)}^{D} l\left(y_{o}, m(y)\right)
$$

$\mathcal{I}$ is defined by the network topology and MAC protocol in use by users of different technologies (cellular/D2D). Interference characterization in stochastic geometry is captured by the location of interferes (using point processes) and the random channel gains $F_{y_{o},(x / y / m(y))}$. Thus, interference can be formulated by its pdf (or its cdf). However, a closed-form expression for the pdf of aggregate interference in large-scale networks is not possible, so, $\mathcal{I}_{a g g}$ is calculated by taking the Laplace transform of the pdf. Alternatively, Characteristic function and moment generation functions are also trivial stochastic geometry tools to characterize $\mathcal{I}_{\text {agg }}$ [6]. In the next section, we evaluate the Laplace transform of aggregate interference at a typical receiver to calculate the coverage probability.

\section{A. Interference Characterization}

The typical node can successfully communicate with the tagged transmitter, if SIR is greater than certain SIR threshold $T$, also known as coverage probability. The success probability is a key parameter which is used to further evaluate expressions for the data rate, throughput and Area Spectral Efficiency (ASE). The probability of success for a typical D2D receiver and BS can be written as,

$$
p_{s}(T) \triangleq \mathbb{P}\left(\operatorname{SIR}_{\mathcal{X}}>T\right)
$$

where, $\mathcal{X}=o^{c}$ when a typical receiver is a cellular BS and $y_{o}$ in case of a typical D2D receiver. First, the interference characterization for a typical D2D receiver is formulated. Using eq. (6) we have

$$
p_{s}^{y_{o}}(T)=\mathbb{P}\left(\frac{P_{D} F_{y_{o}, m_{y_{o}}}^{D} l\left(y_{o}, m_{y_{o}}\right)}{\underset{y_{o}, x}{\mathcal{I}}+\underset{y_{o}, y}{\mathcal{I}}+\underset{y_{o}, m(y) / m_{y_{o}}}{\mathcal{I}}+\beta P_{D} 1_{y, m(y)}^{F D}}>T\right)
$$

The link distance between a typical SU (D2D) and its transmitter is $l\left(y_{o}, m_{y_{o}}\right)$, hence, the distance based path-loss will be $R_{D 2 D}^{-\alpha_{D}}$. Also, simplifying the above expression to apply i.i.d Rayleigh fading with mean 1 , we will have,

$p_{s}^{y_{o}}=\mathbb{P}\left\{F_{y_{o}}^{D}, m_{y_{o}}>T \frac{\mathcal{I}_{o, x}+\mathcal{I}_{y_{o}, y}+\mathcal{I}_{y_{o}, m(y) / m_{y_{o}}}+\beta P_{D} 1_{y, m(y)}^{F D}}{P_{D} R_{D 2 D}^{-\alpha}}\right\}$ 
Considering a i.i.d Rayleigh fading channel, we simplify the above equation for the Laplace transform. As $F_{y_{o}}^{D}, m_{y_{o}}=$ $\exp (1)$ is channel fading/gain during transmission from the tagged transmitter to a typical receiver. Applying the expectation of randomness for PPP and PHP, we have

$p_{s}^{y_{o}}(T)=\mathbb{E}_{\Phi_{c}, \Psi_{D}, \Psi_{m(D)}}\left\{\exp -s\left(\mathcal{I}_{y_{o}, x}+\mathcal{I}_{y_{o}, y}+\mathcal{I}_{y_{o}, m(y) / m_{y_{o}}}+\beta P_{D} 1_{y, m(y)}^{F D}\right)\right\}$

Using the properties of exponential independence of expectation, and converting the summation to a product for the generalized expression,

$p_{s}^{y_{o}}=\exp \left(-s\left(\beta P_{D} 1_{y, m(y)}^{F D}\right) \prod_{\kappa \varepsilon \Phi_{C}, \Psi_{D}, \Psi_{m(D)}} \mathcal{L}_{I_{k}}\left(s \mathcal{I}_{y_{o}, \kappa}\right)\right)$

Laplace transform of interference fields from cellular, HD and FD users will be evaluated in future work along with the validation of the simulation results.

\section{RESUlTS AND Discussions}

According to the system model in Section III, this section presents insightful results from Monte-Carlo Simulations based on the system parameters given in table 1 . The success probability of a typical D2D receiver at the origin is evaluated in different scenarios and thorough discussions on the results are provided. The section presents a performance impact of different LTE transmission types for D2D nodes operating in the $H D$ and $F D$ modes with different $\beta$ values. The network configuration and simulation parameters, notations and values are given in Table I.

Cellular users/EZ intensity and radius: An interesting result which is useful for CBRS systems, is presented in Fig. 4. As the cellular users transmit with comparatively high power and their EZ impact on the D2D link, an increase in $\lambda_{C}$ causes a decrease in $p_{s}$ of a typical user at the origin as shown in Fig. 4 (a). Also, from Eq. 8, the interference field of cellular users $\left(\mathcal{I}_{y_{o}, x}\right)$ increases, causing $S I R_{y_{o}^{D}}$ to fall below the threshold $T$. On the contrary, increasing $R_{E Z}$ (by keeping one cellular user) results in an increase of $p_{s}$. This is due to the fact that higher $R_{E Z}$ will switch more D2D (HD and FD) users inactive due to Interference protection and the cellular user will only be the major interferer. Intuitively, an increase in $R_{E Z}$ decreases $\lambda_{D}$, which results in less interference and higher success probability.

Impact of D2D FD probability, link distance and intensity: Results in Fig. 5 (a),(b) and (c) show the impact of increasing FD users in network $\left(p_{F D}\right)$, increasing the D2D Link distance $\left(R_{D 2 D}\right)$ and increasing the total number of D2D users $\left(\lambda_{D}\right)$, respectively.

In Fig. 5 (a), increased interference due to FD receivers causes perform degradation of a typical user. As represented in Eq. 10, a FD D2D link adds up interference from receivers, hence increasing the overall interference experienced by a typical user. The FD links further adds up interference $\left(\mathcal{I}_{y_{o}, y}\right)$ for a typical user, hence reducing the probability of success. Fig. 5 (b) further explains the impact of shorter and longer

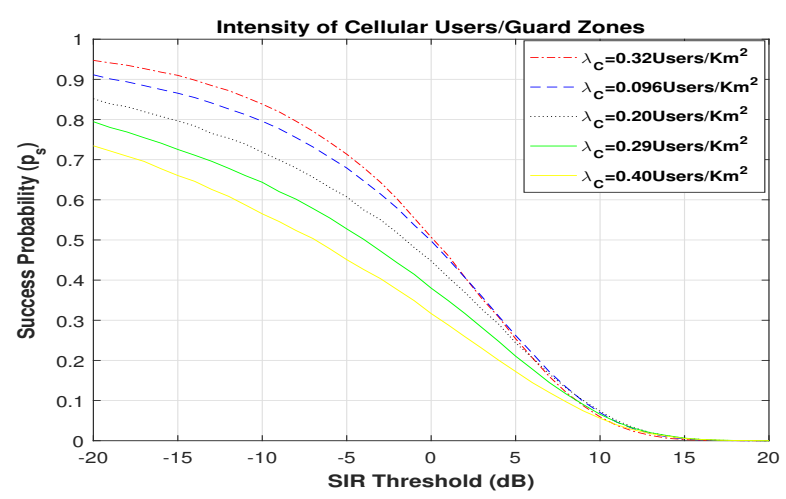

(a)

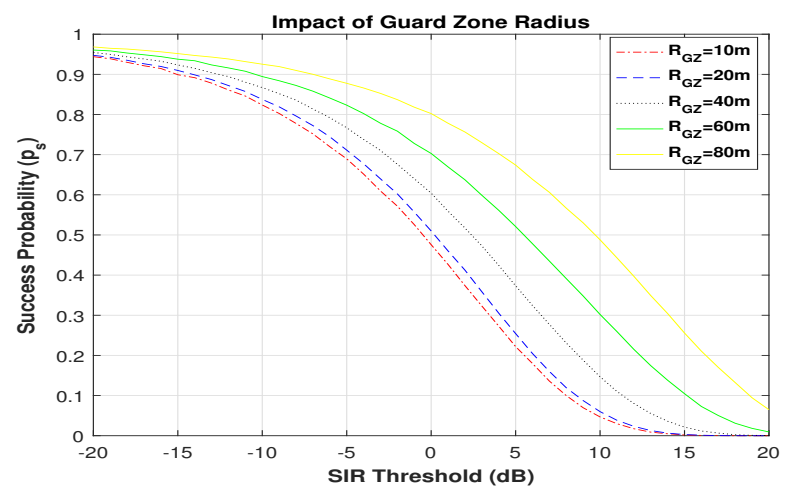

(b)

Fig. 4: Impact of (a) cellular users/EZ intensity and (b) radius of cellular EZ ( $\left.R_{E Z}\right)$ on success probability of typical user

D2D link distances on its performance as also shown in Fig. 3 as the CDF of D2D distance distribution from Eq. 3. Shorter $R_{D 2 D}$ results in $p_{s}$ and vice versa. The shorter D2D links (HD and FD) will add weaker interference components for a typical user. Moreover, the strongest received power will be from the tagged transmitter. The results presented in Fig. 5 (c) validate the analytical expression and also intuitive notion of reduced success probability with an increase in D2D intensity $\left(\lambda_{D}\right)$.

\section{CONCLusion ANd Future Work}

In this article, we have presented stochastic geometry based performance analysis of using FD D2D users while protecting cellular users transmission with exclusion zones. PPP reflects the cellular user distribution, while D2D users reflects a close approximation of PHP. The success probability for a typical D2D user is evaluated for different system parameters, yielding insightful results to capture the performance of Full Duplex technology in D2D while abiding by the interference protection of cellular users. The results have shown the potential of the FD technology, if the D2D link state (HD/FD) is chosen based on aggregate interference. Moreover, an interesting observation based on a shorter D2D link-distance have shown a significant increase in the success probability. 


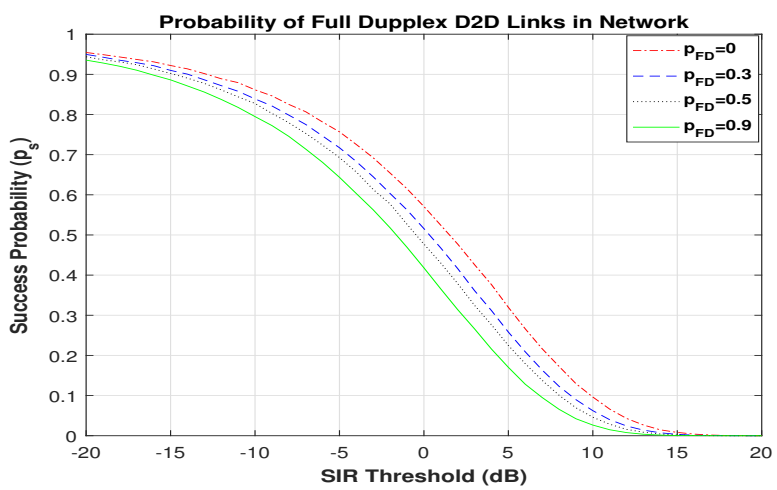

(a)

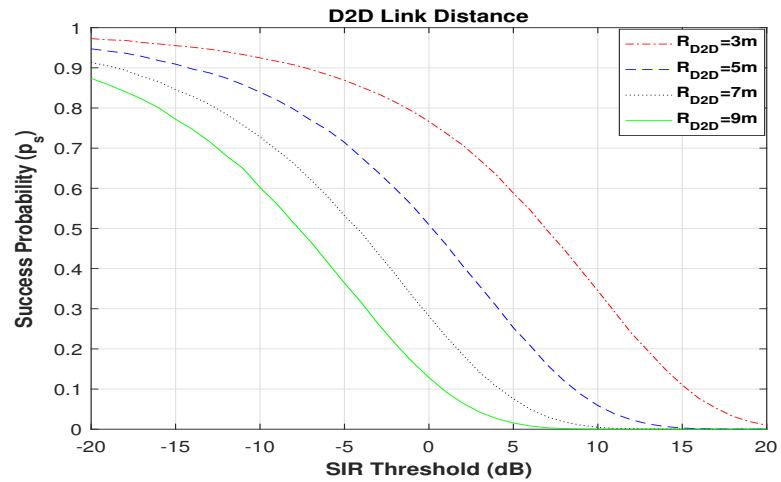

(b)

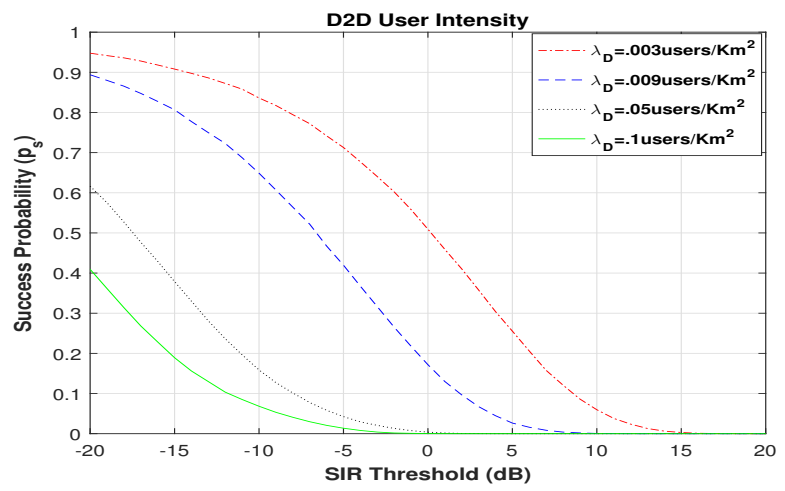

(c)

Fig. 5: Realization of network model with cellular exclusion zone and D2D Links (silent, HD and FD mode)

\section{REFERENCES}

[1] I. F. Akyildiz, W.-Y. Lee, M. C. Vuran, and S. Mohanty, "Next generation/dynamic spectrum access/cognitive radio wireless networks: A survey," Computer Networks, vol. 50, no. 13, pp. 2127 - 2159, 2006.

[2] I. F. Akyildiz, S. Nie, S.-C. Lin, and M. Chandrasekaran, "5g roadmap: 10 key enabling technologies," Computer Networks, vol. 106, pp. 17-48, 2016.

[3] P. Parida, H. S. Dhillon, and P. Nuggehalli, "Stochastic geometry-based modeling and analysis of citizens broadband radio service system," IEEE Access, vol. 5, pp. 7326-7349, 2017.

[4] M. Haenggi, J. G. Andrews, F. Baccelli, O. Dousse, and M. Franceschetti, "Stochastic geometry and random graphs for the analysis and design of wireless networks," IEEE Journal on Selected Areas in Communications, vol. 27, no. 7, pp. 1029-1046, 2009.
[5] J. G. Andrews, A. K. Gupta, and H. S. Dhillon, "A primer on cellular network analysis using stochastic geometry," arXiv preprint arXiv:1604.03183, 2016.

[6] H. ElSawy, E. Hossain, and M. Haenggi, "Stochastic geometry for modeling, analysis, and design of multi-tier and cognitive cellular wireless networks: A survey," Communications Surveys Tutorials askdjfhasdfa, IEEE, vol. 15, no. 3, pp. 996-1019, 2013.

[7] A. Hasan and A. Ali, "Guard zone-based scheduling in ad hoc networks," Computer Communications, vol. 56, pp. 89 - 97, 2015.

[8] Y. Li, F. Baccelli, J. G. Andrews, T. D. Novlan, and J. C. Zhang, "Modeling and analyzing the coexistence of wi-fi and lte in unlicensed spectrum," arXiv preprint arXiv:1510.01392, 2015.

[9] H. S. Dhillon, R. K. Ganti, F. Baccelli, and J. G. Andrews, "Modeling and analysis of k-tier downlink heterogeneous cellular networks," IEEE Journal on Selected Areas in Communications, vol. 30, no. 3, pp. 550560, 2012.

[10] H. Q. Nguyen, F. Baccelli, and D. Kofman, "A stochastic geometry analysis of dense ieee 802.11 networks," in INFOCOM 2007. 26th IEEE International Conference on Computer Communications. IEEE, pp. 1199-1207, IEEE, 2007.

[11] N. Haider, E. Dutkiewicz, D. N. Nguyen, M. Mueck, and S. Srikanteswarae, "The impact on full duplex d2d communication of different lte transmission techniques," in 2017 IEEE 85th Vehicular Technology Conference (VTC Spring), pp. 1-5, June 2017.

[12] M. Naslcheraghi, M. Afshang, and H. S. Dhillon, "Modeling and performance analysis of full-duplex communications in cache-enabled d2d networks," arXiv preprint arXiv:1801.07347, 2018.

[13] Z. Tong and M. Haenggi, "Throughput analysis for full-duplex wireless networks with imperfect self-interference cancellation," IEEE Transactions on Communications, vol. 63, no. 11, pp. 4490-4500, 2015.

[14] K. S. Ali, H. ElSawy, M.-S. Alouini, A. Khavasi, and J. Suk, "Modeling cellular networks with full-duplex d2d communication: A stochastic geometry approach," IEEE Transactions on Communications, vol. 64, no. 10, p. $4409,2016$.

[15] B. A. Jayawickrama, Y. He, E. Dutkiewicz, and M. D. Mueck, "Scalable spectrum access system for massive machine type communication," IEEE Network, pp. 1-7, 2018.

[16] M. Afshang, C. Saha, and H. S. Dhillon, "Nearest-neighbor and contact distance distributions for matérn cluster process," IEEE Communications Letters, vol. 21, no. 12, pp. 2686-2689, 2017.

[17] J. Lee and T. Q. S. Quek, "Device-to-device communication in wireless mobile social networks," in 2014 IEEE 79th Vehicular Technology Conference (VTC Spring), pp. 1-5, May 2014.

[18] M. Haenggi, Stochastic Geometry for Wireless Networks. Cambridge University Press, 2012. 